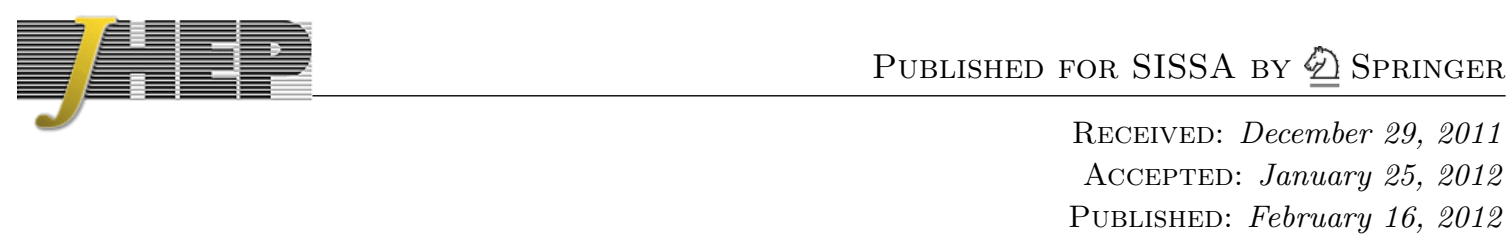

\title{
Twisted masses and enhanced symmetries: the A\&D series
}

\section{Domenico Orlando and Susanne Reffert}

Theory Group, Physics Department, CERN, CH-1211 Geneva 23, Switzerland

\begin{abstract}
We study new symmetries between $A$ and $D$ type quiver gauge theories with different numbers of colors. We realize these gauge theories with twisted masses via a brane construction that reproduces all the parameters of the Gauge/Bethe correspondence.
\end{abstract}

KEYwords: Topological Strings, D-branes, Global Symmetries

ArXIV EPRINT: 1111.4811 


\section{Contents}

1 Introduction 1

2 The $A$ series $\quad 3$

$\begin{array}{lll}3 & \text { The } D \text { series } & 9\end{array}$

4 Dictionary and outlook $\quad 12$

$\begin{array}{ll}\text { A Supersymmetry of the orbifold } & 15\end{array}$

\section{Introduction}

The Gauge/Bethe correspondence [1-3] identifies the supersymmetric vacua of an $\mathcal{N}=$ $(2,2)$ gauge theory in two dimensions with the spectrum of a superselection sector of a Bethe-solvable integrable spin chain. In this note we discuss how the symmetry group of the integrable model is reflected in the gauge theory and we argue that it corresponds to a new global symmetry that relates quiver gauge theories with different numbers of colors.

The action of the symmetry group of the integrable system is exemplified in the sketch in figure 1. The lines represent the energy levels for the eigenstates of an xxx spin chain of length $L=4$ which has symmetry group $\mathrm{SU}(2)$, as a function of the number of magnons $N$. The $A_{1}$ global operators act between states with different values of $N$ preserving the energy (horizontal arrows). This implies that the full spectrum of the chain is organized into SU(2) multiplets (horizontal box). The Gauge/Bethe correspondence identifies the states with a fixed number of magnons (vertical box) with the ground states of a gauge theory (in this case two-dimensional $\mathcal{N}=2^{*} \mathrm{U}(N)$ with $L$ flavors). The $A_{1}$ action thus translates into a symmetry between gauge theories with different numbers of colors. The set of ground states of all the $\mathrm{U}(N)$ gauge theories for $0 \leq N \leq L$ generates the tensor product of $L$ copies of the fundamental representation of $\mathrm{SU}(2)$ [4],

$$
\{\text { ground states }\} \simeq V=\bigotimes_{k=1}^{L} \mathbb{C}^{2} .
$$

Symmetries in $V$ (such as between the $N=1$ and $N=3$ states in figure 1) indicate IR-equivalent gauge theories whose brane realizations are typically related by HananyWitten moves [5]. ${ }^{1}$ In the following, we will study two-dimensional $A_{r}$ and $D_{r}$ type quiver gauge theories which correspond to integrable models with symmetry group $\mathrm{SU}(r+1)$ and $\mathrm{SO}(2 r)$. The raising and lowering operators act on the nodes of the quivers by raising or lowering the number of colors of the corresponding gauge group by one.

\footnotetext{
${ }^{1}$ Different IR equivalences based on the Gauge/Bethe correspondence have been discussed in [6].
} 


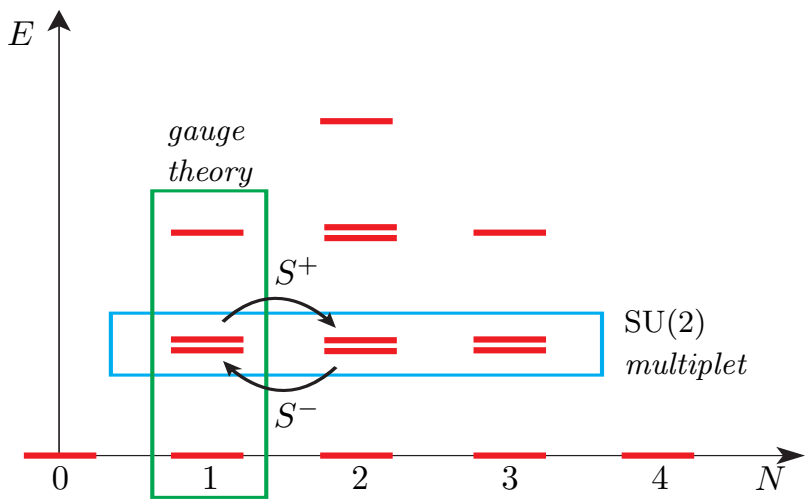

Figure 1. The $A_{1}$ symmetry on the $\mathrm{xxx}_{1 / 2}$ spin chain for $L=4$. The horizontal arrows show the action of the $S^{ \pm}$operators, changing the magnon number $N$, preserving the energy. The spectrum can be organized into multiplets of $\mathrm{SU}(2)$ (horizontal box) or by magnon number (vertical box).

The twisted masses of the gauge fields are a vital ingredient for reproducing the parameters of the integrable system. Schematically, the twisted mass $\widetilde{m}$ for a field $X$ appears in the action as:

$$
\int \mathrm{d}^{4} \theta X^{\dagger} e^{\theta^{-\bar{\theta}^{+}} \widetilde{m}+\text { h.c. }} X \text {. }
$$

The twisted masses are important because they induce an effective twisted superpotential in which a discrete set of normalizable ground states lives.

A mechanism to incorporate these twisted masses into a string theory realization was introduced in [7] for the simplest case corresponding to an $\mathrm{xxx}_{1 / 2}$-spin chain with $\mathrm{SU}(2)$ symmetry. The construction employs a stack of D2-branes suspended between parallel NS5-branes plus a stack of $L$ D4-branes, similar to [5, 8], which are placed into a so-called fluxtrap background which is T-dual to the Melvin or fluxbrane solution [9]. This background also serves to turn on an $\Omega$-background where $\varepsilon_{1}=-\varepsilon_{2} \in \mathbb{R}$ and can be easily generalized to general values of $\varepsilon_{1,2}$ [10]. In this note, we will prove the versatility of this approach by generalizing the mechanism to systems with Yangian symmetry $Y_{\mathrm{SU}(r+1)}$ (i.e. the $A_{r}$ series) and $Y_{\mathrm{SO}(2 r)}$ (i.e. the $D_{r}$ series). While the generalization to the $A_{r}$-case is straightforward and consists in placing $(r+1)$ NS5-branes with stacks of D2-branes between them into the fluxtrap background (section 2), reproducing the $D$ series necessitates the use of a more exotic object, namely an S-dual of an O5-plane on a D5-brane (section 3).

While the symmetry group of the integrable model is not directly manifest in the gauge theory setting, it appears naturally in the string theory construction as an enhanced symmetry for coincident $\mathrm{NS}_{5}$-branes. One of the reasons that this extra symmetry of the gauge theory has been overlooked in the past is that without twisted masses, which are a relatively exotic and therefore rarely used ingredient, the normalizable states on which the symmetry acts in a tractable way disappear.

Before delving into the intricacies of the string theory construction of the Gauge/Bethe correspondence, it is necessary to remind the reader of the parameters of the integrable system we are aiming to reproduce (for a more pedagogical introduction, see [5]). The simplest case of a spin chain consists of a 1d lattice of length $L$ with either an up or a down spin at 
each position $k$. They generate the two-dimensional fundamental representation of SU(2) and, with a proper choice of boundary conditions, the Hamiltonian commutes with the action of a global SU(2). As a result, the full spectrum of the chain can be organized in terms of representations of the symmetry group. In this note, we want to move on to more general systems, in particular those with $A_{r}$ and $D_{r}$ symmetry as opposed to $A_{1}$. A spin chain with a symmetry group of rank $r$ will have $r$ different particle species. For each of them, the chain has an effective length $L_{a}, a=1, \ldots, r . N_{a}$ denotes the number of particles of species $a$ in a given superselection sector. We can furthermore choose a different representation of the symmetry group at each position $k$ in the chain. $\Lambda_{k}^{a}$ denotes the highest weight of the representation at position $k$ for the $a$-th species. On top of that, one can also turn on inhomogeneities at each position of the chain; these are parametrized by $\nu_{k}^{a}$. The eigenvectors of the spin chain are labelled by the rapidities $\lambda_{i}^{(a)}$, which satisfy the Bethe Ansatz equation [11]:

$$
\prod_{k=1}^{L_{a}} \frac{\lambda_{i}^{(a)}+\frac{i}{2}\left(\Lambda_{k}^{a}+\nu_{k}^{a}\right)}{\lambda_{i}^{(a)}-\frac{i}{2}\left(\Lambda_{k}^{a}-\nu_{k}^{a}\right)}=\prod_{\substack{(b, j)=(1,1) \\(b, j) \neq(a, i)}}^{\left(r, N_{b}\right)} \frac{\lambda_{i}^{(a)}-\lambda_{j}^{(b)}+\frac{i}{2} C^{a b}}{\lambda_{i}^{(a)}-\lambda_{j}^{(b)}-\frac{i}{2} C^{a b}}, \quad a=1,2, \ldots, r ; i=1,2, \ldots, N_{a}
$$

where $C^{a b}$ are the elements of the Cartan matrix of the symmetry group. The observation of [1-3] is that the same equations describe the ground states of a corresponding two-dimensional quiver gauge system on a circle (for details see table 4). It is important to note that the twisted masses (which are the counterparts of the parameters $\Lambda, \nu, C$ ) induce an effective twisted superpotential $\widetilde{W}$ whose minima correspond to the normalizable ground states. These ground states are in one-to-one correspondence with the spectrum of the chain and will be identified in the following with BPS configurations in the string theory construction (see [7]).

\section{The $A$ series}

Generalizing the $\mathrm{SU}(2)$ or $A_{1}$ case to $A_{r}$ is very straightforward. We will therefore use this section to remind the reader of the fluxtrap construction [7].

The Cartan matrix of $A_{r}$ series $(\mathrm{SU}(r+1))$ has the form

$$
A=\left(\begin{array}{cccccc}
2 & -1 & 0 & \ldots & & 0 \\
-1 & 2 & -1 & \ldots & & 0 \\
0 & -1 & 2 & -1 & \ldots & 0 \\
\vdots & & \ddots & \ddots & \ddots & \vdots \\
0 & 0 & \ldots & -1 & 2 & -1 \\
0 & 0 & \ldots & 0 & -1 & 2
\end{array}\right)
$$

which gives rise to the Dynkin diagram in figure $2 \mathrm{a}$. The $a$-th node of the Dynkin diagram translates directly to the $a$-th node of the quiver gauge theory. Its color group $\mathrm{U}\left(N_{a}\right)$ is determined by the superselection sector; the length parameter fixes the flavor group $\mathrm{U}\left(L_{a}\right)$ which is attached to the $a$-th node by fundamental and antifundamental fields $Q_{k}^{a}, \bar{Q}_{k}^{a}$, 


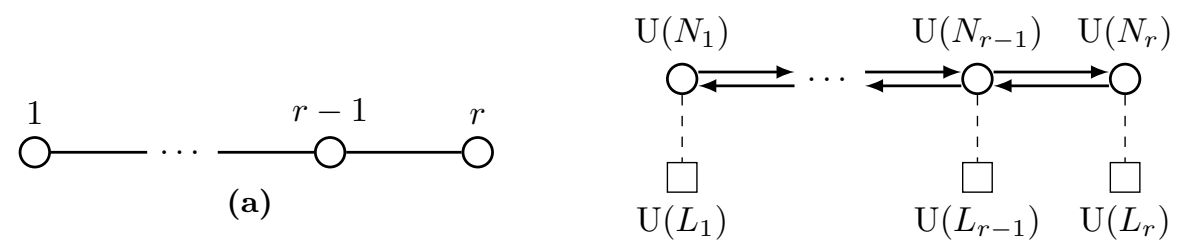

(b)

Figure 2. Dynkin diagram (a) and quiver diagram (b) for the $A_{r}$ series. In the quiver diagram, nodes are gauge groups and squares flavor groups. Each arrow represents a bifundamental and each dotted line a pair fundamental-antifundamental. Each node carries also an adjoint field (not represented).

\begin{tabular}{|c|c|c|c|c|c|c|c|c|c|c|c|}
\hline & & 0 & 1 & 2 & 3 & 4 & 5 & 6 & 7 & 8 & 9 \\
\hline & fluxbrane & $x$ & $x$ & $x$ & $x$ & & & & & & $x$ \\
\hline & NS5 & $x$ & $x$ & & & & & $x$ & $x$ & $x$ & $x$ \\
\hline & D2 & $x$ & $x$ & $x$ & & & & & & & \\
\hline $\mathcal{N}=(2,2)$ & $\mathrm{D} 4$ & $\times$ & $\times$ & & $\times$ & $x$ & $x$ & & & & \\
\hline $\mathcal{N}=(1,1)$ & D4' & $x$ & $x$ & & $x$ & & & & & $x$ & $x$ \\
\hline
\end{tabular}

Table 1. Type IIA Brane configuration for the $2 \mathrm{~d} \mathcal{N}=(2,2)$ and $\mathcal{N}=(1,1)$ theories living on the $\mathrm{D} 2$ branes and reproducing the $A$ series symmetry. The crosses in the fluxbrane row mark the directions in which no identifications are imposed.

$k=1, \ldots L_{a}$. Each arrow between nodes $a$ and $b$ of the quiver diagram corresponds to a bifundamental field $B^{a, b}$ of $\mathrm{U}\left(N_{a}\right) \times \mathrm{U}\left(N_{b}\right)$. Moreover, each node can carry an adjoint field $\Phi^{a}$ (not pictured).

Brane construction. One can construct the $A_{r}$ quiver gauge theory in type IIA string theory by placing $r+1$ parallel $\mathrm{NS}_{5}$-branes extended in the 16789 directions into a flat background [12] (see figure 5 and table 1). When the $\mathrm{NS}_{5}$ s coincide, the six-dimensional $\mathcal{N}=(2,0)$ type IIA theory acquires a $\mathrm{SU}(r+1)$ symmetry that acts on the tensionless strings. Imposing periodic boundary conditions for $x_{1}$, we can T-dualize and obtain a system of coincident $\mathrm{NS}_{5}$-branes in type IIB, which now has standard $\mathrm{SU}(r+1)$ gauge symmetry $^{2}[13]$. This is the enhanced $\mathrm{SU}(r+1)$ symmetry that acts on the ground states of the effective theory of the probe D2-branes.

Suspended between the $a$-th and $(a+1)$-st $\mathrm{NS}_{5}$-brane is a stack of $N_{a}$ D2-branes extended in the 12 directions. The motions of the open strings within the $a$-th stack of D2-branes in the 67 directions are described by the adjoint fields $\Phi^{a}$, while the strings going from the $a$-th to the $(a+1)$-st stack correspond to the bifundamental fields $B$. This configuration preserves eight supersymmetries, which correspond to an $\mathcal{N}=(4,4)$ gauge theory in two dimensions. Gauge invariance and supersymmetry fix the superpotential

\footnotetext{
${ }^{2}$ The periodicity of $x_{1}$ translates into periodic boundary conditions for the two-dimensional gauge theory. This is necessary in order to reproduce the Bethe Ansatz equations.
} 


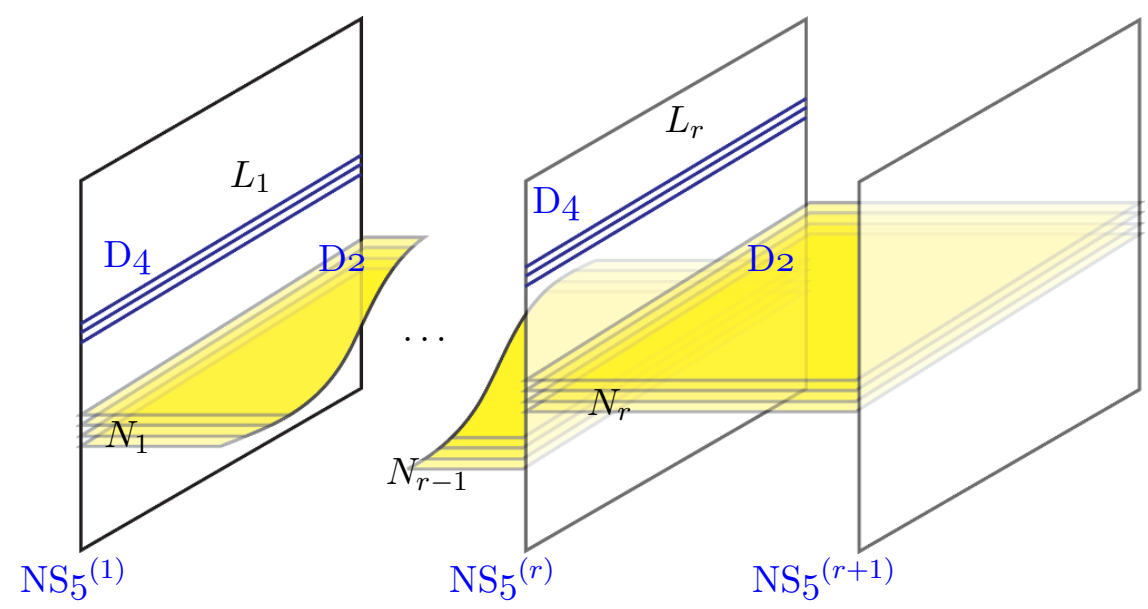

Figure 3. Brane cartoon for the $A_{r}$ model.

couplings of adjoints and bifundamentals to

$$
W=\sum_{a=1}^{r-1} B^{a, a+1} \Phi^{a+1} B^{a+1, a}-B^{a+1, a} \Phi^{a} B^{a, a+1} .
$$

The flavor groups live on stacks of $L_{a}$ D4-branes. They can be added in two different ways:

1. D4'-branes extended in the 1389 directions break half of the supersymmetry and lead to an $\mathcal{N}=(2,2)$ gauge theory on the D2-branes. In this case, the D4-branes can be made to coincide with one of the $\mathrm{NS}_{5}$ in the $x_{2}$ direction in which the $\mathrm{NS}_{5}$-branes are separated, and broken in two. We can then distinguish upper-half D4'-branes $\left(x_{3}>0\right)$ and lower-half D4'-branes $\left(x_{3}<0\right)$. The strings from the D2-branes to the upper-half D4'-branes are described by fundamental fields $Q_{k}^{a}$ and those going to the lower-half D4'-branes by antifundamental fields $\bar{Q}_{k}^{a}$.

2. $\mathrm{D}_{4}$-branes extended in the 1345 directions preserve the same $\mathcal{N}=(4,4)$ supersymmetry as the $\mathrm{NS}_{5}-\mathrm{D}_{2}$ system. In this case, gauge invariance and supersymmetry require the presence of a superpotential term

$$
W=\sum_{k=1}^{L_{a}} Q_{k}^{a} \Phi^{a} \bar{Q}_{k}^{a}
$$

Twisted masses. To reproduce the parameters of the integrable system, twisted masses need to be turned on for all fields, which reduces the supersymmetry of the system by one half.

The twisted masses of the adjoint and bifundamental fields do not originate from the brane construction, but can be turned on by placing the above construction into a so-called fluxtrap background [7], which we will detail in the following. For the moment we observe 
that the superpotential term coupling bifundamentals and adjoints in eq. (2.2) preserves a $\mathrm{U}(1)$ symmetry

$$
e^{i m}:\left(B^{a+1, a}, \Phi^{a}, B^{a, a+1}\right) \mapsto\left(e^{-i m / 2} B^{a+1, a}, e^{i m} \Phi^{a}, e^{-i m / 2} B^{a, a+1}\right),
$$

which constrains the corresponding twisted masses to obey

$$
\widetilde{m}^{\mathrm{b}(a+1, a)}+\widetilde{m}^{\mathrm{adj}(a)}+\widetilde{m}^{\mathrm{b}(a, a+1)}=0 .
$$

A twisted mass $m$ for the adjoints $\Phi^{a}$ automatically requires twisted masses for the bifundamentals that can be gauge-fixed to $-m / 2$.

For the fundamentals and antifundamentals, the two cases above must again be distinguished.

1. In the case of $\mathcal{N}=(2,2)$ supersymmetry broken to $\mathcal{N}=(1,1)$, twisted masses can be turned on by moving the upper-half and lower-half $\mathrm{D}_{4}$ '-branes away from each other. Their $x_{6}+i x_{7}$-values are the twisted masses for the $Q_{k}^{a}, \bar{Q}_{k}^{a}$-fields [8]. In particular, the relative separation of the upper-half and lower-half $\mathrm{D}_{4}$ ' corresponds to the $\Lambda_{k}^{a}$ parameter from the spin chain, while the center of mass of the position corresponds to the $\nu_{k}^{a}$ parameter.

2. In the case of $\mathcal{N}=(4,4)$ supersymmetry broken to $\mathcal{N}=(2,2)$, the representations of the symmetry group at each position of the chain is restricted to $\Lambda_{k}^{a}=1$ or $\Lambda_{k}^{a}=0$, while it is impossible to turn on impurities, thus $\nu_{k}^{a}=0$. The twisted masses of the fundamental and antifundamental fields are inherited directly from the fluxtrap background via the superpotential term $\sum_{k=1}^{L_{a}} Q_{k}^{a} \Phi^{a} \bar{Q}_{k}^{a}$ that we have encountered in eq. (2.3), following the same mechanism outlined above for the twisted masses of the bifundamentals (see [7]).

The fluxbrane background. Let us concentrate on the $\mathrm{NS}_{5}-\mathrm{D}_{2}$ system. In order to understand the fluxtrap background and the corresponding twisted masses for the fields $\Phi$ and $B$ it is convenient to consider the T-dual type IIB setup in which $\mathrm{NS}_{5}$ s are extended in the 16789 directions and $\mathrm{D}_{3}$-branes are extended in the 128 directions.

The fluxbrane construction is obtained by imposing a set of identifications $\Gamma$ on the space orthogonal to the $\mathrm{D}_{3}$-branes. The directions 3 and 9 will be spectators. Let $x_{8}$ be periodic with radius $\widetilde{R}$,

$$
x_{8}=\widetilde{R} \widetilde{u}, \quad \widetilde{u} \simeq \widetilde{u}+2 \pi k_{1},
$$

and the remaining four directions 4567 be described by cylindrical coordinates $\left(\rho_{1}, \theta_{1}, \rho_{2}, \theta_{2}\right)$. The space $\mathbb{R}^{5} / \Gamma$ is obtained by imposing

$$
\left\{\begin{array}{l}
\widetilde{u} \simeq \widetilde{u}+2 \pi k_{1}, \\
\theta_{1} \simeq \theta_{1}+2 \pi m \widetilde{R} k_{1}, \quad k_{1} \in \mathbb{Z}, \\
\theta_{2} \simeq \theta_{2}-2 \pi m \widetilde{R} k_{1},
\end{array}\right.
$$

in addition to the standard identifications for cylindrical coordinates,

$$
\theta_{1} \simeq \theta_{1}+2 \pi k_{2}, \quad \theta_{2} \simeq \theta_{2}+2 \pi k_{3}, \quad k_{2}, k_{3} \in \mathbb{Z} .
$$


In order to disentangle the periodicities we introduce the new angular variables

$$
\left\{\begin{array}{l}
\phi_{1}=\theta_{1}-m \widetilde{R} \widetilde{u} \\
\phi_{2}=\theta_{2}+m \widetilde{R} \widetilde{u}
\end{array}\right.
$$

which are $2 \pi$-periodic.

Since the $\mathrm{NS}_{5}$ s are much heavier than the $\mathrm{D}_{3}$-branes, we can treat the latter as probes propagating in the background generated by the backreaction of the former. In rectilinear coordinates $\left(x_{4}+i x_{5}=\rho_{1} e^{i \phi_{1}}, x_{6}+i x_{7}=\rho_{2} e^{i \phi_{2}}\right)$ the fields in the bulk read:

$$
\begin{aligned}
\widetilde{\mathrm{d} s}^{2}= & -\mathrm{d} x_{0}^{2}+\mathrm{d} x_{1}^{2}+\mathrm{d} x_{8}^{2}+\mathrm{d} x_{9}^{2}+U\left[\mathrm{~d} x_{2}^{2}+\mathrm{d} x_{3}^{2}+\sum_{i=4}^{5}\left(\mathrm{~d} x_{i}+m V^{i} \mathrm{~d} x_{8}\right)^{2}\right] \\
& +\sum_{i=6}^{7}\left(\mathrm{~d} x_{i}+m V^{i} \mathrm{~d} x_{8}\right)^{2} \\
\mathrm{~d} B= & * \mathrm{~d} U=b_{i} \mathrm{~d} x^{i} \wedge\left(\mathrm{d} \phi_{1}+m \widetilde{R} \mathrm{~d} \widetilde{u}\right) \\
\Phi= & \log \left(\widetilde{R} g_{3}^{2}\right)+\frac{1}{2} \log U
\end{aligned}
$$

where

$$
\begin{aligned}
U & =1+\sum_{a=1}^{r+1} \frac{\alpha^{\prime}}{\left(x_{2}-X_{2}^{(a)}\right)^{2}+\left(x_{3}-X_{3}^{(a)}\right)^{2}+x_{4}^{2}+x_{5}^{2}}, \\
V^{i} \partial_{i} & =-x^{5} \partial_{x_{4}}+x^{4} \partial_{x_{5}}+x^{7} \partial_{x_{6}}-x^{6} \partial_{x_{7}}=\partial_{\phi_{1}}-\partial_{\phi_{2}},
\end{aligned}
$$

and $\left(X_{2}^{(a)}, X_{3}^{(a)}\right)$ is the position of the $a$-th $\mathrm{NS}_{5}$. This provides the $\Omega$-deformation of the NS5 background. Note that the $\mathrm{NS}_{5}$-branes are forced to sit in $X_{3}^{(a)}=X_{4}^{(a)}=0$ in order to preserve the rotation symmetry in the $\left(x_{4}, x_{5}\right)$ plane that is necessary for the fluxbrane construction.

Now we can T-dualize in the direction $\widetilde{u}$ to go back to our original $\mathrm{NS}_{5}-\mathrm{D}_{2}$ configuration. The bulk fields in the $\mathrm{NS}_{5}$-fluxtrap background are given by

$$
\begin{aligned}
\mathrm{d} s^{2}= & -\mathrm{d} x_{0}^{2}+\mathrm{d} x_{1}^{2}+U\left[\mathrm{~d} x_{2}^{2}+\mathrm{d} x_{3}^{2}+\mathrm{d} x_{4}^{2}+\mathrm{d} x_{5}^{2}\right]+\mathrm{d} x_{6}^{2}+\mathrm{d} x_{7}^{2}+\mathrm{d} x_{9}^{2}, \\
& +\frac{1}{\Delta^{2}}\left[\left(m b_{i} \mathrm{~d} x^{i}+\mathrm{d} x_{8}\right)^{2}-m^{2}\left(U\left(x_{4} \mathrm{~d} x_{5}-x_{5} \mathrm{~d} x_{4}\right)-x_{6} \mathrm{~d} x_{7}+x_{7} \mathrm{~d} x_{6}\right)^{2}\right], \\
B= & \frac{1}{\Delta^{2}}\left[b_{i} \mathrm{~d} x^{i} \wedge\left(\mathrm{d} \phi_{1}+m^{2} \rho_{2}^{2}\left(\mathrm{~d} \phi_{1}+\mathrm{d} \phi_{2}\right)\right)+m\left(U \rho_{1}^{2} \mathrm{~d} \phi_{1}-\rho_{2}^{2} \mathrm{~d} \phi_{2}\right) \wedge \mathrm{d} x_{8}\right], \\
e^{-\Phi}= & \frac{1}{g_{3}^{2} \sqrt{\alpha^{\prime}}} \frac{\Delta}{\sqrt{U}},
\end{aligned}
$$

where

$$
\Delta^{2}=1+m^{2}\left(U \rho_{1}^{2}+\rho_{2}^{2}\right)
$$

The $\mathrm{NS}_{5}$-fluxtrap background preserves eight supercharges. The conserved Killing spinors have been found in [7] and read

$$
\left\{\begin{array}{l}
\epsilon_{L}=e^{-\Phi / 8}\left(\nVdash+\Gamma_{11}\right) \Pi_{-}^{\mathrm{NS} 5} \Pi_{-}^{\text {flux }} \exp \left[\frac{1}{2} \phi_{1} \Gamma_{45}+\frac{1}{2} \phi_{2} \Gamma_{67}+\frac{1}{2} \phi_{3} \Gamma_{23}\right] \epsilon_{0}, \\
\epsilon_{R}=e^{-\Phi / 8}\left(\nVdash-\Gamma_{11}\right) \Gamma_{u} \Pi_{+}^{\mathrm{NS} 5} \Pi_{-}^{\mathrm{flux}} \exp \left[\frac{1}{2} \phi_{1} \Gamma_{45}+\frac{1}{2} \phi_{2} \Gamma_{67}+\frac{1}{2} \phi_{3} \Gamma_{23}\right] \epsilon_{1},
\end{array}\right.
$$


where $\epsilon_{0}$ and $\epsilon_{1}$ are constant Majorana spinors,

$$
\Gamma_{u}=\frac{m \rho_{1}}{\Delta} \Gamma_{5}-\frac{m \rho_{2}}{\Delta} \Gamma_{7}+\frac{1}{\Delta} \Gamma_{8}
$$

and

$$
\Pi_{ \pm}^{\text {flux }}=\frac{1}{2}\left(\nVdash \pm \Gamma_{4567}\right), \quad \quad \Pi_{ \pm}^{\mathrm{NS}_{5}}=\frac{1}{2}\left(\nVdash \pm \Gamma_{2345}\right)
$$

Other parameters. At this point we can identify the geometric origin of the remaining gauge theory parameters.

- The gauge couplings $e_{a}$ for the $\mathrm{U}\left(N_{a}\right)$ theories are given by the separations of the $\mathrm{NS}_{5}$-branes in the $x_{2}$ direction (the direction in which the D2s have a finite extension):

$$
\left(X_{2}^{(a+1)}-X_{2}^{(a)}\right) \frac{\ell_{\mathrm{st}}}{g_{\mathrm{st}}}=\frac{1}{e_{a}^{2}},
$$

where $\ell_{\text {st }}$ is the string length and $g_{\text {st }}$ is the type IIA string coupling constant.

- The Fayet-Iliopoulos (FI) parameters are proportional to the separations of the $\mathrm{NS}_{5}$-branes in the $x_{3}$ direction: $^{3}$

$$
\frac{X_{3}^{(a+1)}-X_{3}^{(a)}}{\ell_{\mathrm{st}} g_{\mathrm{st}}}=-\tau_{a} .
$$

The $\theta$ angle does not have a direct geometric interpretation in type IIA, but requires the uplift to M-theory.

Both a non-vanishing gauge coupling and a non-vanishing FI parameter break the $A_{r}$ symmetry, albeit in very different ways.

- When the gauge coupling vanishes, we conjecture that all the massive states decouple, thus leaving only the $A_{r}$-charged ground states. ${ }^{4}$ The new massive states appearing for non-zero gauge coupling do not have an analogue on the integrable system side. This is why the coupling $e_{a}$ in eq. (2.22) does not correspond to a parameter of the spin chain.

- A non-vanishing Fi parameter does not add new states, but breaks the $A_{r}$ symmetry. This corresponds directly to imposing a non-periodic boundary condition on the chain. The Hilbert space remains the same, but the Hamiltonian does not commute anymore with the generators of the global $A_{r}$. The FI parameter acts as a twist that splits up the energy levels of all $A_{r}$ multiplets (see [14]).

\footnotetext{
${ }^{3}$ In the $\mathcal{N}=(4,4)$ case broken to $\mathcal{N}=(2,2)$, the directions 3,4 and 5 are initially equivalent, which corresponds to the three independent FI parameters of the $\mathcal{N}=(4,4)$ theory. This symmetry is broken by the fluxtrap that fixes the $x_{4}$ and $x_{5}$ positions of the $\mathrm{NS}_{5}$ s to the origin, thus leaving the single FI parameter of $\mathcal{N}=(2,2)$.

${ }^{4}$ This argument does not exclude the presence of $A_{r}$-charged BPS-protected states, whose existence is currently under investigation.
} 
Ground states as BPS states. The ground states of the D2 probe branes extended in the directions 1 and 2 can be understood as 1/2-BPS objects in the NS5-fluxbrane background (four supercharges). As expected, the effect of the fluxtrap is that the minimum energy configurations are localized at the bottom of a potential at $x_{3}=x_{4}=x_{5}=0$. More general BPS states with less supersymmetry can be constructed, but in general they are not localized and hence non-normalizable. A DBI analysis around the stable configurations shows that in the effective gauge theory description, all the adjoint fields $\Phi^{a}$ which describe the motion in the directions 6 and 7 receive the same twisted mass term $m$. This mass is propagated to the bifundamental fields $B^{a, a+1}$ via the superpotential coupling as explained above.

We have thus constructed a brane realization of the full $A_{r}$ quiver gauge theory which includes all the parameters of the corresponding integrable model, in particular the twisted masses. The symmetry group of the spin chain is reflected in the symmetry of the background containing the parallel $\mathrm{NS}_{5}$-branes. The Gauge/Bethe correspondence implies the action of the background $A_{r}$ symmetry on the probe branes such that the raising and lowering operators $E_{a}^{ \pm}$change the gauge group at the $a$-th node:

$$
E_{a}^{ \pm}: \mathrm{U}\left(N_{a}\right) \mapsto U\left(N_{a} \pm 1\right)
$$

\section{The $D$ series}

The Cartan matrix of the $D_{r}$ series $(\mathrm{SO}(2 r))$ has the form

$$
A=\left(\begin{array}{ccccccc}
2 & -1 & 0 & \ldots & & & 0 \\
-1 & 2 & -1 & \ldots & & & 0 \\
0 & -1 & 2 & -1 & & & 0 \\
\vdots & & \ddots & \ddots & \ddots & & \vdots \\
0 & 0 & \ldots & & 2 & -1 & -1 \\
0 & 0 & \ldots & & -1 & 2 & 0 \\
0 & 0 & \ldots & & -1 & 0 & 2
\end{array}\right),
$$

which gives rise to the Dynkin diagram in figure 4a.

Looking at the Dynkin diagram, we see that up to the second last node, it is the same as the one of the $A_{r}$ series. Up to this node we can therefore replicate the brane construction of the last section, consisting of parallel NS5-branes with stacks of D2-branes between them. Let us concentrate on the nodes $r-2, r-1$ and $r$. From the Dynkin diagram, we learn that there should be no bifundamental fields between the last two nodes, but extra bifundamental fields between nodes $r-2$ and $r$. To achieve this, we need to make use of a somewhat more exotic object, which is best described as the S-dual of a D5-brane coincident with an O5-plane [15-19]. For further convenience, we will name this object NO5. In our picture, it corresponds to a $\mathbb{Z}_{2}$ orbifold $\mathcal{I}_{4}$ which reflects the 2345 directions times the action of $(-1)^{F_{L}}$, where $F_{L}$ is the left-moving fermion number. The $\mathrm{NO}_{5}$ orbifold preserves exactly the same supersymmetries as an $\mathrm{NS}_{5}$-brane extended in the 16789 directions as discussed in appendix A. 


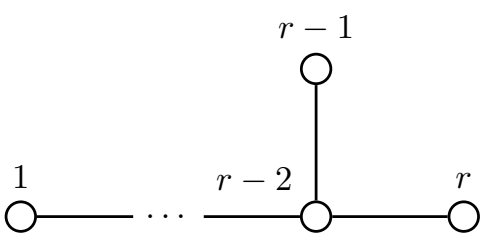

(a)

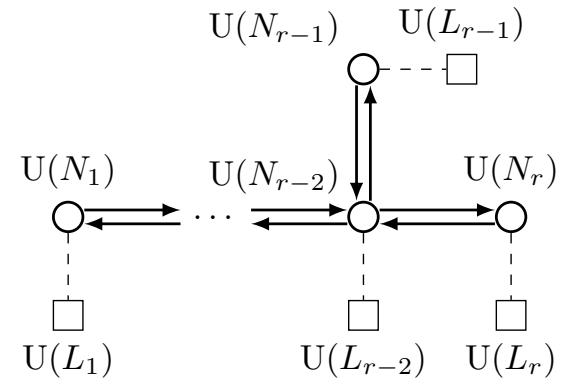

(b)

Figure 4. Dynkin diagram and quiver diagram for $D_{r}$ series. In the quiver diagram, nodes are gauge groups and squares flavor groups. Each arrow represents a bifundamental and each dotted line a pair fundamental-antifundamental. Each node carries also an adjoint field (not represented).

\begin{tabular}{|c|c|c|c|c|c|c|c|c|c|c|c|}
\hline & & 0 & 1 & 2 & 3 & 4 & 5 & 6 & 7 & 8 & 9 \\
\hline & NS5 & $\times$ & $x$ & & & & & $x$ & $x$ & $x$ & $\times$ \\
\hline & NO5 & $x$ & $x$ & & & & & $x$ & $x$ & $x$ & $\times$ \\
\hline & D3 & $\times$ & $x$ & $x$ & & & & & & $x$ & \\
\hline $\mathcal{N}=4$ & D5 & $x$ & $x$ & & $x$ & $x$ & $x$ & & & $x$ & \\
\hline $\mathcal{N}=2$ & D3' & $x$ & $\times$ & & $x$ & & & & & & $\times$ \\
\hline
\end{tabular}

Table 2. Type IIB brane configuration for the $3 \mathrm{~d} \mathcal{N}=4$ and $\mathcal{N}=2$ theories living on the $\mathrm{D}_{3}$ branes. The crosses in the $\mathrm{NO}_{5}$ orbifold row mark the directions in which the space remains flat and no identifications are imposed.

Field content. As for the fluxbrane construction, it is convenient to start from the T-dual configuration in which $\mathrm{D}_{3}$-branes are suspended between $\mathrm{NS}_{5}$-branes and the $\mathrm{NO}_{5}$ orbifold. $^{5}$ Consider a stack of $N_{2}+N_{3}$ D 3 -branes extended in the 128 directions, suspended between the NO5-plane localized at $x_{2}=x_{3}=x_{4}=x_{5}=0$ and an NS5-brane localized at $\left(x_{2}=X_{2}^{(2)}, x_{3}=X_{3}^{(2)}, x_{4}=0, x_{5}=0\right)$ (see table 2).

The six-dimensional theory living on the $\mathrm{NO}_{5}$ orbifold has $\mathrm{SO}(2)$ symmetry, under which the $\mathrm{D}_{3}$-branes are magnetically charged. This means that in the stack of $\mathrm{D}_{3}$ s we can distinguish the $N_{3}$ positively charged $\mathrm{D}_{3}{ }^{+}$from the $N_{2}$ negatively charged $\mathrm{D}_{3}{ }^{-}$. The $\mathrm{U}\left(N_{2}+N_{3}\right)$ symmetry is broken to $\mathrm{U}\left(N_{2}\right) \times \mathrm{U}\left(N_{3}\right)$, as one can verify by writing the partition function for open strings between two $\mathrm{D}_{3}$-branes: strings between two branes with the same $\mathrm{SO}(2)$ charge and strings between $\mathrm{D}_{3}{ }^{+}$and $\mathrm{D}_{3}{ }^{-}$behave differently under the inversion of coordinates and do not mix. From the effective gauge theory point of view, the oscillations of the $\mathrm{D}_{3}$-branes transverse to the $\mathrm{NO}_{5}$ result in adjoint fields for both $\mathrm{U}\left(N_{2}\right)$ and $\mathrm{U}\left(N_{3}\right)$, while the bifundamentals are projected out by the action of $\mathcal{I}_{4}$ (see [17]).

At this point we can add a second parallel $\mathrm{NS}_{5}$-brane at $x_{2}=X_{2}^{(1)}, x_{3}=X_{3}^{(1)}$ and a stack of $N_{1} \mathrm{D}_{3}$-branes suspended between the two $\mathrm{NS}_{5}$-branes. The configuration around

\footnotetext{
${ }^{5}$ The $\mathrm{NO}_{5}$ remains invariant under T-duality in a parallel direction [17].
} 


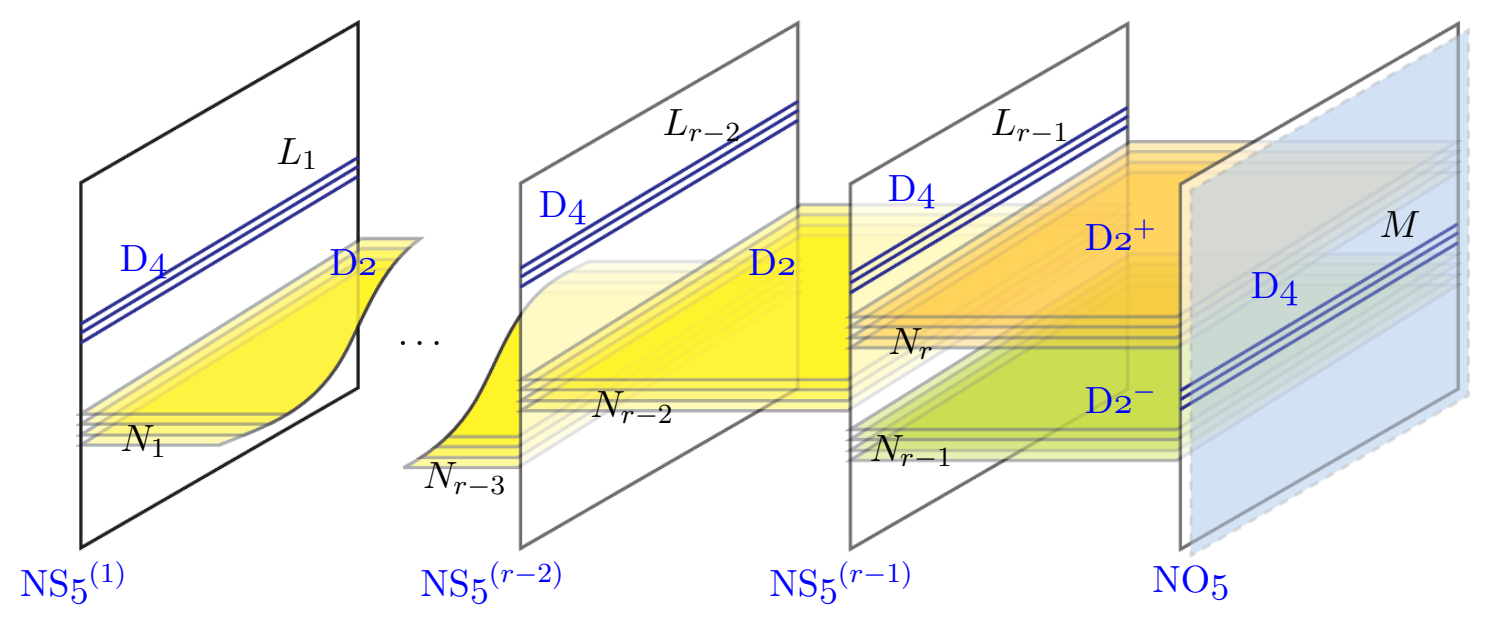

Figure 5. Brane cartoon for the $D_{r}$ model.

the $\mathrm{NS}_{5}$ at $\left(X_{2}^{(2)}, X_{3}^{(2)}\right)$ is the same that we have encountered in the previous section. This means that together with the adjoints of $\mathrm{U}\left(N_{1}\right)$ coming from the $\mathrm{D}_{3}$-branes on the left, we also have bifundamentals for $\mathrm{U}\left(N_{1}\right) \times \mathrm{U}\left(N_{2}\right)$ and $\mathrm{U}\left(N_{1}\right) \times \mathrm{U}\left(N_{3}\right)$ which are coupled via a $\sum_{a=2}^{3}\left(B^{a, 1} \Phi^{1} B^{1, a}-B^{1, a} \Phi^{a} B^{a, 1}\right)$ term. The field content of the theory is thus summarized by a quiver diagram with $D_{3}$ shape (see figure $4 \mathrm{~b}$ ). Higher $D_{r}$ diagrams are obtained by adding more parallel $\mathrm{NS}_{5}$ s with $\mathrm{D}_{3}$-branes suspended in between (see the cartoon in figure 5).

Following the construction presented in the previous section, we can add fundamentals to the two-dimensional gauge theory by inserting more branes into the system in two ways:

1. breaking half of the supersymmetry such that the effective theory has $\mathcal{N}=2$ supersymmetry in three dimensions (four supercharges). In this case we add a stack of $\mathrm{D}_{3}$ '-branes extended in the 139 directions that end on the $\mathrm{NS}_{5}$ and can be split into an upper and lower part (extended in $x_{3} \gtrless 0$ );

2. respecting the same supersymmetries preserved by the $\mathrm{NO}_{5}-\mathrm{NS}_{5}-\mathrm{D}_{3}$ system. In this case, the effective gauge theory has $\mathcal{N}=4$ supersymmetry in three dimensions (eight supercharges). This corresponds to having stacks of $\mathrm{D}_{5}$-branes extended in the 13458 directions and living in the intervals between the $\mathrm{NS}_{5}$-branes.

The new possibility with respect to the $A_{r}$ configuration is that a stack of $M \mathrm{D}_{3}$ '-branes or $\mathrm{D}_{5}$-branes can end on the $\mathrm{NO}_{5}$ plane. In this case, the corresponding open strings give rise to $L_{r}=2 M+L_{r-1}$ fundamentals for the $\mathrm{U}\left(N_{r}\right)$ node (see [18]).

Fluxbrane, fluxtrap and twisted masses. Now that we know that the gauge theory describing the oscillations of D3-branes in the $\mathrm{NO}_{5}-\mathrm{NS}_{5}-\mathrm{D}_{5}$ system has the right matter content to reproduce the $D$ series Bethe Ansatz equations, we need to verify that the orbifold action is compatible with the fluxbrane construction that gives rise to the necessary twisted masses. 
Following the previous section we start from the T-dual type IIB $\mathrm{NO}_{5}$-NS5 system and introduce polar coordinates $\left(\rho_{1}, \theta_{1}, \rho_{2}, \theta_{2}\right)$ for four of the directions perpendicular to the $\mathrm{D}_{3}$-branes. The two identifications that we want to impose are

- the orbifold action which reflects $\rho_{1} e^{i \theta_{1}}$, leaving $\rho_{2} e^{i \theta_{2}}$ invariant, and reflects $x_{2}, x_{3}$, which are spectators for the fluxbrane:

$$
\mathcal{I}_{4}:\left(x_{2}, x_{3}, \rho_{1}, \theta_{1}, \rho_{2}, \theta_{2}\right) \mapsto\left(-x_{2},-x_{3}, \rho_{1}, \theta_{1}+\pi, \rho_{2}, \theta_{2}\right) .
$$

- the fluxbrane identifications on flat space,

$$
\left\{\begin{array}{l}
\widetilde{u} \simeq \widetilde{u}+2 \pi k_{1} \\
\theta_{1} \simeq \theta_{1}+2 \pi m \widetilde{R} k_{1}, \\
\theta_{2} \simeq \theta_{2}-2 \pi m \widetilde{R} k_{1},
\end{array}\right.
$$

which are disentangled by introducing the angles

$$
\phi_{1}=\theta_{1}-m \widetilde{R} \widetilde{u}, \quad \phi_{2}=\theta_{2}+m \widetilde{R} \widetilde{u} .
$$

We need to verify that it is possible to define the action of $\mathcal{I}_{4}$ on the coordinates $\phi_{i}$, which automatically implement the fluxbrane identifications. Using the definition of $\phi_{1}$ and $\phi_{2}$, it is immediate to see that the orbifold action remains simple,

$$
\mathcal{I}_{4}:\left(x_{2}, x_{3}, \rho_{1}, \phi_{1}, \rho_{2}, \phi_{2}, \widetilde{u}\right) \mapsto\left(-x_{2},-x_{3}, \rho_{1}, \phi_{1}+\pi, \rho_{2}, \phi_{2}, \widetilde{u}\right) .
$$

We conclude thus that the orbifold action is compatible with the fluxbrane construction and it is formally the same as in flat space when written for the disentangled coordinates.

At this point we can T-dualize the system in the direction $x_{8}$ and obtain the $\mathrm{NO}_{5}-\mathrm{NS}_{5}$-fluxtrap background in which the movement of the $\mathrm{D}_{2}$-branes is described by a $\mathcal{N}=(2,2)$ two-dimensional gauge theory with a twisted mass term $m$ for the adjoints. Under T-duality, both the $\mathrm{D}_{3}$ '-branes and the $\mathrm{D}_{5}$-branes turn into $\mathrm{D}_{4}$-branes. In the $\mathcal{N}=(2,2)$ case, the respective fundamentals and antifundamentals acquire a twisted mass $-m / 2$ via the superpotential coupling as in eq. (2.5). In the $\mathcal{N}=(1,1)$ case, generic twisted masses can be obtained by breaking the $\mathrm{D}_{4}$ '-branes into an upper and a lower part (see the configuration outlined in table 3). The identification of the remaining parameters is the same as in the previous section.

Once more, the Gauge/Bethe correspondence implies an action of the $\mathrm{SO}(2 r)$ group on the quiver gauge theories that we have realized. Raising and lowering operators $E_{a}^{ \pm}$ change the number of colors at the corresponding nodes by one as in eq. (2.24).

\section{Dictionary and outlook}

In this note we have extended the brane construction of the gauge system corresponding to the $\mathrm{XXx}_{1 / 2}$ model [7] to the general case of $A_{r}$ and $D_{r}$ spin chains. The main novelty of this construction is the inclusion of twisted masses into the quiver gauge theories. The 


\begin{tabular}{|c|c|c|c|c|c|c|c|c|c|c|c|}
\hline & & 0 & 1 & 2 & 3 & 4 & 5 & 6 & 7 & 8 & 9 \\
\hline & fluxbrane & $x$ & $x$ & $x$ & $x$ & & & & & & $x$ \\
\hline & $\mathrm{NS}_{5}$ & $x$ & $x$ & & & & & $x$ & $x$ & $x$ & $x$ \\
\hline & $\mathrm{NO}_{5}$ & $\times$ & $x$ & & & & & $x$ & $x$ & $x$ & $\times$ \\
\hline & $\mathrm{D}_{2}$ & $\times$ & $\times$ & $\times$ & & & & & & & \\
\hline $\mathcal{N}=(2,2)$ & $\mathrm{D}_{4}$ & $x$ & $x$ & & $x$ & $x$ & $x$ & & & & \\
\hline $\mathcal{N}=(1,1)$ & $\mathrm{D}_{4}^{\prime}$ & $x$ & $x$ & & $x$ & & & & & $x$ & $x$ \\
\hline
\end{tabular}

Table 3. Type IIA Brane configuration for the $2 \mathrm{~d} \mathcal{N}=(2,2)$ and $\mathcal{N}=(1,1)$ theories living on the $\mathrm{D}_{2}$-branes and reproducing the $D$ series symmetry. The crosses in the fluxbrane and $\mathrm{NO}_{5}$ rows mark the directions in which no identification is imposed.

twisted masses introduce a potential for the ground states which can be identified via the Gauge/Bethe correspondence - with the eigenvectors of a spin chain. Our main result is that the enhanced background symmetry of the $\mathrm{NS}_{5}$ (or $\mathrm{NO}_{5}-\mathrm{NS}_{5}$ ) system acts on the states of the gauge theories that describe the motion of the probe D2-branes.

The results of the previous two sections are summarized in a dictionary, see table 4 . It is based on the Gauge/Bethe dictionary table given in [5], with a new column containing the translation to the parameters of the brane constructions for the $A_{r}$ and $D_{r}$ series. As already discussed, we are indeed able to match all the parameters of the integrable system/gauge theory to parameters in the string theory construction. Note that in table 4 we assume the more general case of $\mathcal{N}=(1,1)$ supersymmetry, where the D4-branes are ending on the $\mathrm{NS}_{5}$. In the more supersymmetric $\mathcal{N}=(2,2)$ configuration, which corresponds to the fundamental representation on all positions and no inhomogeneities in the spin chain, the $\mathrm{D}_{4} \mathrm{~s}$ are differently oriented and are located in the intervals between two $\mathrm{NS}_{5}$-branes (as discussed in [5]). In this case the twisted masses of the fundamental and anti-fundamental fields are determined uniquely by the fluxtrap parameter $m$ which gets propagated to these fields via the superpotential term $Q \Phi \bar{Q}$, which is absent in the $\mathcal{N}=(1,1)$ case.

Of course, the question of how to generalize this brane construction further is of importance as the possible symmetry groups of the integrable systems captured by the Gauge/Bethe correspondence go beyond what was covered in this note. The most obvious candidates are the $B, C$ and $E$ series, but also supergroup symmetries are possible. Unfortunately, these examples are far more challenging. Quiver gauge theories are finite if and only if the quiver is an affine Dynkin diagram of type $A, D$, or $E$ [20]. This spells trouble for the $B$ and $C$ series. The approach followed here seems also not suitable to capture the exceptional groups. Studying the T-dual ALE singularities may be a promising alternative venue, see [21].

The generalization of the string theory construction to all symmetry groups appearing in Bethe-solvable integrable systems will thus remain a topic of further investigation for some time to come. 


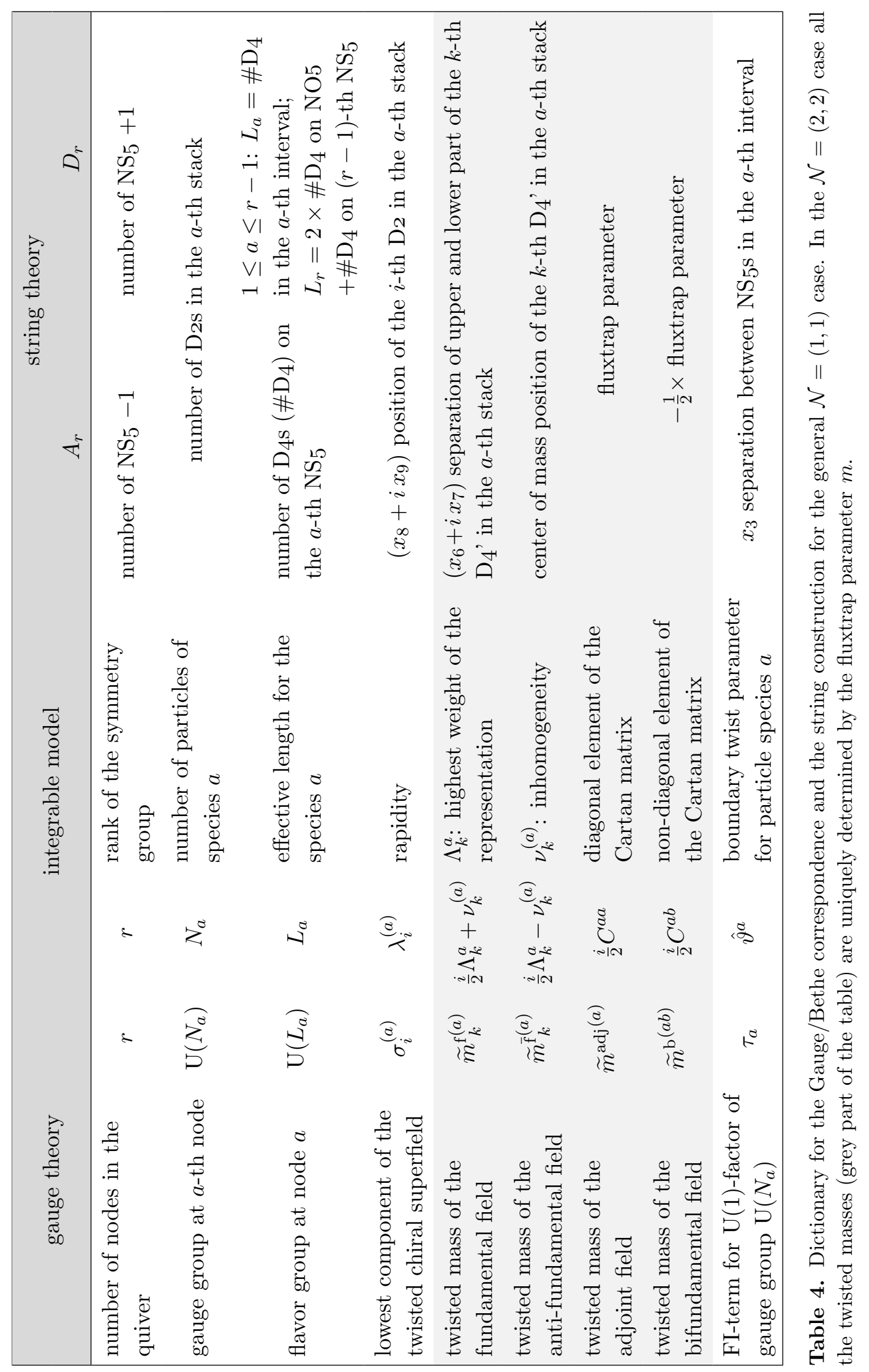




\section{Acknowledgments}

It is our pleasure to thank Simeon Hellerman and Wolfgang Lerche for enlightening discussions and comments on the manuscript, and Davide Forcella for correspondence.

\section{A Supersymmetry of the orbifold}

In this appendix we derive the Killing spinors preserved by the $\mathrm{NO}_{5}-\mathrm{NS}_{5}$-fluxbrane background.

In section 3 we have seen that the fluxbrane identifications are compatible with the $\mathrm{NO}_{5}$ action. This can be written as $\mathcal{I}_{4} \cdot(-1)^{F_{L}}$ where $\mathcal{I}_{4}$ is the inversion $\mathcal{I}_{4}:\left(x_{2}, x_{3}, x_{4}, x_{5}\right) \mapsto\left(-x_{2},-x_{3},-x_{4},-x_{5}\right)$ and $F_{L}$ is the left-moving fermion number. The space is locally flat but in order to write the orbifold action on the Killing spinors it is convenient to use polar coordinates:

$$
x_{4}+i x_{5}=\rho_{1} e^{i \phi_{1}}, \quad x_{6}+i x_{7}=\rho_{2} e^{i \phi_{2}}, \quad x_{2}+i x_{3}=\rho_{3} e^{i \phi_{3}} .
$$

The solutions to the type IIB Killing spinor equations for the fluxbrane background in these coordinates are given by [7]

$$
K^{\text {flux }}=\Pi_{-}^{\text {flux }} \exp \left[\frac{1}{2} \phi_{1} \Gamma_{45}+\frac{1}{2} \phi_{2} \Gamma_{67}+\frac{1}{2} \phi_{3} \Gamma_{23}\right] \epsilon_{0} \equiv \epsilon_{L}^{\text {flux }}+\epsilon_{R}^{\text {flux }},
$$

where $\epsilon_{0}$ is a constant complex Weyl spinor and $\Pi^{\text {flux }}$ is the projector that keeps only the spinors compatible with the fluxbrane identifications:

$$
\Pi_{ \pm}^{\text {flux }}=\frac{1}{2}\left(\nVdash \pm \Gamma_{4567}\right) .
$$

These are the spinors that satisfy the local equations and the fluxbrane identifications, now we have to impose the orbifold boundary conditions. The effect of $\mathcal{I}_{4}$ on the polar coordinates is

$$
\mathcal{I}_{4}:\left(\rho_{1}, \phi_{1}, \rho_{2}, \phi_{2}, \rho_{3}, \phi_{3}\right) \mapsto\left(\rho_{1}, \pi+\phi_{1}, \rho_{2}, \phi_{2}, \rho_{3}, \pi+\phi_{3}\right)
$$

Using the fact that

$$
\exp \left[\frac{\pi}{2}\left(\Gamma_{23}+\Gamma_{45}\right)\right]=\Gamma_{2345},
$$

and adding the sign coming from the $(-1)^{F_{L}}$ factor, we find that the orbifold acts as

$$
\mathcal{I}_{4} \cdot(-1)^{F_{L}}:\left(\begin{array}{c}
\epsilon_{L}^{\text {flux }} \\
\epsilon_{R}^{\text {flux }}
\end{array}\right) \mapsto\left(\begin{array}{cc}
-\Gamma_{2345} & \\
& \Gamma_{2345}
\end{array}\right)\left(\begin{array}{l}
\epsilon_{L}^{\text {flux }} \\
\epsilon_{R}^{\text {flux }}
\end{array}\right) .
$$

At this point we need to project out the Killing spinors that are not invariant under the action of the orbifold. This is obtained by introducing the projectors

$$
\Pi_{ \pm}^{\mathrm{NO} 5}=\frac{1}{2}\left(\nVdash \pm \Gamma_{2345}\right)
$$


which satisfy

$$
\Gamma_{2345} \Pi_{ \pm}^{\mathrm{NO} 5}= \pm \Pi_{ \pm}^{\mathrm{NO} 5}
$$

so that the 8 orbifold-invariant Killing spinors can be written as

$$
\left\{\begin{array}{l}
\epsilon_{L}^{N O 5}=\Pi_{-}^{\text {NO5 }} \epsilon_{L}^{\text {flux }}=\Pi_{-}^{\text {NO5 }} \Pi_{-}^{\text {flux }} \exp \left[\frac{1}{2} \phi_{1} \Gamma_{45}+\frac{1}{2} \phi_{2} \Gamma_{67}+\frac{1}{2} \phi_{3} \Gamma_{23}\right] \epsilon_{0}, \\
\epsilon_{R}^{N O 5}=\Pi_{+}^{\text {NO5 }} \epsilon_{R}^{\text {flux }}=\Pi_{+}^{\text {NO5 }} \Pi_{-}^{\text {flux }} \exp \left[\frac{1}{2} \phi_{1} \Gamma_{45}+\frac{1}{2} \phi_{2} \Gamma_{67}+\frac{1}{2} \phi_{3} \Gamma_{23}\right] \epsilon_{1},
\end{array}\right.
$$

Note that $\Pi_{ \pm}^{\text {NO5 }}$ are precisely the same projectors $\Pi_{ \pm}^{\text {NS5 }}$ that appears for $\mathrm{NS}_{5}$-branes oriented in the same directions (see eq. (2.21)).

T-duality in $x_{8}$ translates this result into the IIA orbifolded fluxtrap picture where the Killing spinors $K^{I I A}=\epsilon_{L}+\epsilon_{R}$ are:

$$
\left\{\begin{array}{l}
\epsilon_{L}=e^{-\Phi / 8}\left(\nVdash+\Gamma_{11}\right) \Pi_{-}^{\mathrm{NO} 5} \Pi_{-}^{\text {flux }} \exp \left[\frac{1}{2} \phi_{1} \Gamma_{45}+\frac{1}{2} \phi_{2} \Gamma_{67}+\frac{1}{2} \phi_{3} \Gamma_{23}\right] \epsilon_{0} \\
\epsilon_{R}=e^{-\Phi / 8}\left(\nVdash-\Gamma_{11}\right) \Gamma_{u} \Pi_{+}^{\mathrm{NO} 5} \Pi_{-}^{\text {flux }} \exp \left[\frac{1}{2} \phi_{1} \Gamma_{45}+\frac{1}{2} \phi_{2} \Gamma_{67}+\frac{1}{2} \phi_{3} \Gamma_{23}\right] \epsilon_{1}
\end{array}\right.
$$

where $\epsilon_{0}$ and $\epsilon_{1}$ are constant Majorana spinors,

$$
\Gamma_{u}=\frac{m \rho_{1}}{\Delta} \Gamma_{5}-\frac{m \rho_{2}}{\Delta} \Gamma_{7}+\frac{1}{\Delta} \Gamma_{8}
$$

and

$$
\Delta^{2}=1+m^{2}\left(U \rho_{1}^{2}+\rho_{2}^{2}\right) .
$$

Open Access. This article is distributed under the terms of the Creative Commons Attribution License which permits any use, distribution and reproduction in any medium, provided the original author(s) and source are credited.

\section{References}

[1] N.A. Nekrasov and S.L. Shatashvili, Quantum integrability and supersymmetric vacua, Prog. Theor. Phys. Suppl. 177 (2009) 105 [arXiv:0901.4748] [InSPIRE].

[2] N.A. Nekrasov and S.L. Shatashvili, Supersymmetric vacua and Bethe ansatz, Nucl. Phys. Proc. Suppl. 192-193 (2009) 91 [arXiv:0901.4744] [INSPIRE].

[3] N.A. Nekrasov and S.L. Shatashvili, Quantization of integrable systems and four dimensional gauge theories, arXiv:0908.4052 [INSPIRE].

[4] D. Orlando and S. Reffert, The Gauge-Bethe correspondence and geometric representation theory, Lett. Math. Phys. 98 (2011) 289 [arXiv:1011.6120] [INSPIRE].

[5] D. Orlando and S. Reffert, Relating gauge theories via Gauge/Bethe correspondence, JHEP 10 (2010) 071 [arXiv: 1005.4445] [inSPIRE].

[6] H.-Y. Chen, N. Dorey, T.J. Hollowood and S. Lee, A new $2 d / 4 d$ duality via integrability, JHEP 09 (2011) 040 [arXiv: 1104.3021] [INSPIRE].

[7] S. Hellerman, D. Orlando and S. Reffert, String theory of the Omega deformation, JHEP 01 (2012) 148 [arXiv:1106.0279] [INSPIRE].

[8] A. Hanany and K. Hori, Branes and $N=2$ theories in two-dimensions, Nucl. Phys. B 513 (1998) 119 [hep-th/9707192] [INSPIRE]. 
[9] M. Melvin, Pure magnetic and electric geons, Phys. Lett. 8 (1964) 65 [InSPIRE].

[10] S. Reffert, General Omega deformations from closed string backgrounds, arXiv:1108.0644 [INSPIRE].

[11] N.Y. Reshetikhin, The spectrum of the transfer matrices connected with Kac-Moody algebras, Lett. Math. Phys. 14 (1987) 235.

[12] A. Hanany and E. Witten, Type IIB superstrings, BPS monopoles and three-dimensional gauge dynamics, Nucl. Phys. B 492 (1997) 152 [hep-th/9611230] [INSPIRE].

[13] E. Witten, Some comments on string dynamics, hep-th/9507121 [INSPIRE].

[14] P. Kulish and A. Stolin, Deformed yangians and integrable models, Czechoslovak J. Phys. 47 (1997) 1207 [q-alg/9708024].

[15] A. Sen, Duality and orbifolds, Nucl. Phys. B 474 (1996) 361 [hep-th/9604070] [INSPIRE].

[16] A. Sen, Stable non-BPS bound states of BPS D-branes, JHEP 08 (1998) 010 [hep-th/9805019] [INSPIRE].

[17] A. Kapustin, $D_{n}$ quivers from branes, JHEP 12 (1998) 015 [hep-th/9806238] [INSPIRE].

[18] A. Hanany and A. Zaffaroni, Issues on orientifolds: on the brane construction of gauge theories with $\mathrm{SO}(2 N)$ global symmetry, JHEP 07 (1999) 009 [hep-th/9903242] [INSPIRE].

[19] S. Hellerman, New type-II string theories with sixteen supercharges, hep-th/0512045 [INSPIRE].

[20] S. Katz, P. Mayr and C. Vafa, Mirror symmetry and exact solution of $4 D N=2$ gauge theories: 1, Adv. Theor. Math. Phys. 1 (1998) 53 [hep-th/9706110] [INSPIRE].

[21] W. Lerche and N. Warner, Exceptional SW geometry from ALE fibrations, Phys. Lett. B 423 (1998) 79 [hep-th/9608183] [INSPIRE]. 